\title{
Some novelties on intersecting families of subsets
}

\author{
Gyula O. H. Katona
}

\begin{abstract}
A family $\mathcal{F}$ of subsets of an $n$-element set $[n]$ is called intersecting if any two members have a non-empty intersection. The theorem of Erdős, Ko and Rado claims that an intersecting family of $k$-element subsets of $[n]$ has at most $\left(\begin{array}{l}n-1 \\ k-1\end{array}\right)$ members if $2 k \leq n$. That is, the best is to choose all sets containing a fixed element. Such a family is called trivially intersecting. An old generalization of this theorem is due to Frankl where $[n]$ is partitioned into two disjoint parts: $[n]=X_{1} \cup X_{2}$ and the members of the family have $k$ elements in $X_{1}$ and $\ell$ elements in $X_{2}$, respectively. The largest intersecting family is trivially intersecting, again. We show a further generalization, where a set of pairs of integers, $\left(k_{i}, \ell_{i}\right)$ is given and the members of the family have $k_{i}$ elements in $X_{1}$ and $\ell_{i}$ elements in $X_{2}$, respectively, for some $i$. The shadow $\sigma(\mathcal{F})$ of a family $\mathcal{F}$ of $k$-element subsets is a family of all $k$-1-element sets obtained by deleting single elements from the members. A sharp lower bound was known for $|\sigma(\mathcal{F})|$ in terms of $|\mathcal{F}|$ under the condition that the family is $t$-intersecting, that is, when any two members have at least $t$ elements in common. The extremal construction, however, has a small number of members,
\end{abstract}

2000 AMS Subject Classification: 05D05.

Key Words and Phrases: intersecting family, two-part family, shadow.

This research was supported by the National Research, Development and Innovation Office - NKFIH Fund No's SSN117879, NK104183 and K116769. 
depending only on $k$ and $t$. We show improvements for the case when the number of members is a polynomial function of $n$.

\section{Introduction}

The underlying set will be $\{1,2, \ldots, n\}$. The family of all $k$-element subsets of $[n]$ is denoted by $\left(\begin{array}{c}{[n]} \\ k\end{array}\right)$. Its subfamilies are called uniform. A family $\mathcal{F}$ of some subsets of $[n]$ is called intersecting if $F \cap G \neq \emptyset$ holds for every pair $F, G \in \mathcal{F}$. Erdős, Ko and Rado started to look for the largest intersecting families on $n$ elements in 1936. They did not publish it until 1961 since they thought it was not interesting for the general public. Now this paper [4] is one of the most cited papers of Erdős, although there is a strong competition. Their first observation was that if all sizes are allowed then one can choose at most $2^{n-1}$ subsets, since a set and its complement cannot be simultaneously chosen. The family containing a fixed element shows that this estimate is sharp. However, the main result of [4] determines the largest intersecting family consisting of subsets of size exactly $k$, that is the case of uniform families. The problem is trivial when $k>\frac{n}{2}$ : all $k$-element subsets can be chosen. It is not so trivial at all when $k \leq \frac{n}{2}$.

Theorem 1. (Erdős, Ko, Rado [4]) If $\mathcal{F} \subset\left(\begin{array}{c}{[n]} \\ k\end{array}\right)$ is intersecting where $k \leq \frac{n}{2}$ then

$$
|\mathcal{F}| \leq\left(\begin{array}{l}
n-1 \\
k-1
\end{array}\right)
$$

Does this result belong to the main direction of the conference? Where are the cliques?

The Kneser graph $K(n, k)$ is a graph with vertex set $V=\left(\begin{array}{c}{[n]} \\ k\end{array}\right)$, two vertices $A, B \in V$ are adjacent iff $A \cap B=\emptyset$. Using this terminology we can restate Theorem 1:

Theorem 2. (Erdös, Ko, Rado [4]) Suppose $k \leq \frac{n}{2}$. Then the independence number $\alpha(K(n, k))$ (that is the size of the largest empty subgraph) 
is

$$
\left(\begin{array}{l}
n-1 \\
k-1
\end{array}\right)
$$

Of course, the clique number of the complement of $K(n, k)$ is the same. A small example is $K(5,2)$, the Petersen graph: $\alpha(K(5,2))=\left(\begin{array}{l}4 \\ 1\end{array}\right)=4$. While the size of the largest clique in $\overline{K(5,2)}$ is $\omega(\overline{K(5,2)})=\left(\begin{array}{l}4 \\ 1\end{array}\right)=4$.

The original proof of the Erdős-Ko-Rado theorem uses the so called shifting method. There is a shorter proof based on the cycle method in [12]. It can also be found in the books [1] and [2]. If $k<\frac{n}{2}$ there is only one extremal construction.

Construction 1. Take all subsets of $[n]$ having size $k$ and containing the element 1.

In the case when $k=\frac{n}{2}$ there are many extremal constructions.

Construction 2. If $k=\frac{n}{2}$ one can choose one from each complementing pair, freely.

We say that a family $\mathcal{F}$ is trivially intersecting if there is an element $a \in[n]$ such that all members of $\mathcal{F}$ contain $a$. Construction 1 is trivially intersecting, Construction 2 not necessarily. Paper [4] posed the problem of finding the largest $k$-uniform non-trivially intersecting family. It was found by Hilton and Milner.

Theorem 3. [9] If $\mathcal{F}$ is an intersecting but not a trivially intersecting family, $\mathcal{F} \subset\left(\begin{array}{c}{[n]} \\ k\end{array}\right)(2 k \leq n)$ then

$$
|\mathcal{F}| \leq 1+\left(\begin{array}{c}
n-1 \\
k-1
\end{array}\right)-\left(\begin{array}{c}
n-k-1 \\
k-1
\end{array}\right)
$$

The construction giving equality is the following.

Construction 3. Let $K=\{2,3, \ldots, k+1\}$. The extremal family will consist of $K$ and all $k$-element sets containing 1 and intersecting $K$.

Let me call the reader's attention to the new book of Gerbner and Patkós [8], containg many related results. 


\section{Two-part intersecting families}

Now we will consider the problem when the underlying set is partitioned into two parts $X_{1}, X_{2}$ and the sets $F \in \mathcal{F}$ have fixed sizes in both parts. For some motivation see [13] (Section 4). More precisely let $X_{1}$ and $X_{2}$ be disjoint sets of $n_{1}$, respectively $n_{2}$ elements. Paper [6] considered such subsets of $X=X_{1} \cup X_{2}$ which had $k$ elements in $X_{1}$ and $\ell$ elements in $X_{2}$. The family of all such sets is denoted by

$\left(\begin{array}{c}X_{1}, X_{2} \\ k, \ell\end{array}\right)=\left(\begin{array}{c}X_{1} \\ k\end{array}\right) \biguplus\left(\begin{array}{c}X_{2} \\ \ell\end{array}\right)=\left\{F \subset X_{1} \cup X_{2}:\left|F \cap X_{1}\right|=k,\left|F \cap X_{2}\right|=\ell\right\}$.

The construction above, taking all possible sets containing a fixed element also works here. If the fixed element is in $X_{1}$ then the number of these sets is

$$
\left(\begin{array}{c}
n_{1}-1 \\
k-1
\end{array}\right)\left(\begin{array}{c}
n_{2} \\
\ell
\end{array}\right)
$$

otherwise it is

$$
\left(\begin{array}{c}
n_{1} \\
k
\end{array}\right)\left(\begin{array}{c}
n_{2}-1 \\
\ell-1
\end{array}\right)
$$

The following theorem of Frankl [6] claims that the larger one of these is the best.

Theorem 4. [6] Let $X_{1}, X_{2}$ be two disjoint sets of $n_{1}$ and $n_{2}$ elements, respectively. The positive integers $k, \ell$ satisfy the inequalities $2 k \leq n_{1}, 2 \ell \leq$ $n_{2}$. If $\mathcal{F}$ is an intersecting subfamily of $\left(\begin{array}{c}X_{1}, X_{2} \\ k, \ell\end{array}\right)$ then

$$
|\mathcal{F}| \leq \max \left\{\left(\begin{array}{c}
n_{1}-1 \\
k-1
\end{array}\right)\left(\begin{array}{c}
n_{2} \\
\ell
\end{array}\right),\left(\begin{array}{c}
n_{1} \\
k
\end{array}\right)\left(\begin{array}{c}
n_{2}-1 \\
\ell-1
\end{array}\right)\right\} .
$$

Actually his theorem is formulated for an arbitrary number of parts.

Theorem 4 could be formulated in such a way that the largest subfamily of (1) is one of the trivially intersecting families. It is natural to ask what is the largest non-trivially intersecting subfamily. 
Take a Hilton-Milner family (Construction 3 ) in $X_{1}$, denote it by $\operatorname{HM}\left(X_{1}, k\right)$. Extend its members in all possible ways by $\ell$-element subsets chosen from $X_{2}$ :

$$
\operatorname{HM}_{1}\left(X_{1}, k ; X_{2}, \ell\right)=\left\{F \cup G: F \in \operatorname{HM}\left(X_{1}, k\right), G \subset X_{2},|G|=\ell\right\} .
$$

Define, similarly,

$$
\operatorname{HM}_{2}\left(X_{1}, k ; X_{2}, \ell\right)=\left\{F \cup G: F \subset X_{1},|F|=k, G \in \operatorname{HM}\left(X_{2}, \ell\right)\right\} .
$$

It was conjectured in [13] that either $\mathrm{HM}_{1}\left(X_{1}, k ; X_{2}, \ell\right)$ or $\mathrm{HM}_{2}\left(X_{1}, k ; X_{2}, \ell\right)$ is the largest nontrivially intersecting subfamily of $\left(\begin{array}{c}X_{1}, X_{2} \\ k, \ell\end{array}\right)$. Kwan, Sudakov and Vieira [16] showed that this is not true: there are other, "mixed" Hilton-Milner families which are better in some cases.

Fix an element $a \in X_{1}$, a set $A \subset X_{1}$ such that $a \notin A,|A|=k$ and a set $B \subset X_{2}$ such that $|B|=\ell$ and define

$\operatorname{HM}_{1}^{\operatorname{mix}}\left(X_{1}, k ; X_{2}, \ell\right)=\left\{F:\left|F \cap X_{1}\right|=k,\left|F \cap X_{2}\right|=\ell, a \in F, F \cap(A \cup B) \neq \emptyset\right\}$.

$\operatorname{HM}_{2}^{\operatorname{mix}}\left(X_{1}, k ; X_{2}, \ell\right)$ is the symmetric construction.

Theorem 5. (Kwan, Sudakov, Vieira [16]) If both $\left|X_{1}\right|$ and $\left|X_{2}\right|$ are large enough then the largest non-trivially intersecting subfamily of $\left(\begin{array}{c}X_{1}, X_{2} \\ k, \ell\end{array}\right)$ is one of

$\operatorname{HM}_{1}\left(X_{1}, k ; X_{2}, \ell\right), \operatorname{HM}_{2}\left(X_{1}, k ; X_{2}, \ell\right), \operatorname{HM}_{1}^{\operatorname{mix}}\left(X_{1}, k ; X_{2}, \ell\right) \quad$ and $\operatorname{HM}_{2}^{\operatorname{mix}}\left(X_{1}, k ; X_{2}, \ell\right)$.

Their result actually claims the analogous statement for more parts. The proof uses the shifting method.

Suppose now the case when two sizes are also allowed in both parts (but not independently!) that is the family consists of sets satisfying $\left|F \cap X_{1}\right|=k,\left|F \cap X_{2}\right|=\ell$ or $\left|F \cap X_{1}\right|=r,\left|F \cap X_{2}\right|=s$. Using the notation above, we will consider intersecting subfamilies of

$$
\left(\begin{array}{c}
X_{1}, X_{2} \\
k, \ell
\end{array}\right) \bigcup\left(\begin{array}{c}
X_{1}, X_{2} \\
r, s
\end{array}\right) .
$$


In Theorem 3

$$
\left(\begin{array}{c}
n_{1}-1 \\
k-1
\end{array}\right)\left(\begin{array}{c}
n_{2} \\
\ell
\end{array}\right) \geq\left(\begin{array}{c}
n_{1} \\
k
\end{array}\right)\left(\begin{array}{c}
n_{2}-1 \\
\ell-1
\end{array}\right)
$$

holds if and only if

$$
\frac{k}{n_{1}}\left(\begin{array}{c}
n_{1} \\
k
\end{array}\right)\left(\begin{array}{c}
n_{2} \\
\ell
\end{array}\right) \geq \frac{\ell}{n_{2}}\left(\begin{array}{c}
n_{1} \\
k
\end{array}\right)\left(\begin{array}{c}
n_{2} \\
\ell
\end{array}\right)
$$

that is when

$$
\frac{k}{\ell} \geq \frac{n_{1}}{n_{2}} .
$$

In this case the best is a trivially intersecting family with fixing one point on the left hand side. Otherwise the point should be fixed on the right hand side. Of course the same holds for the pair $r, s$ therefore if

$$
\frac{k}{\ell}, \frac{r}{s} \geq \frac{n_{1}}{n_{2}}
$$

then the best, for both kinds of sets, is to fix one point on the left hand side.

But what happens if

$$
\frac{k}{\ell}>\frac{n_{1}}{n_{2}}>\frac{r}{s} ?
$$

For the family of sets having $k$ and $\ell$ elements in the two sizes, respectively, the best construction chooses the fixed element on the left hand side, for the other family on the right hand side. These two families together are not intersecting. The answer to our question is that one of them wins! That is if both $n_{1}$ and $n_{2}$ are large then the largest intersecting family is trivially intersecting, either on the left or on the right hand side.

Let us consider now the more general case when other sizes are also allowed, that is, the family consists of sets satisfying $\left|F \cap X_{1}\right|=k_{i}, \mid F \cap$ $X_{2} \mid=\ell_{i}$ for certain pairs $\left(k_{i}, \ell_{i}\right)$ of positive integers. Using the notation above, we will consider subfamilies of

$$
\bigcup_{i=1}^{m}\left(\begin{array}{c}
X_{1}, X_{2} \\
k_{i}, \ell_{i}
\end{array}\right) \text {. }
$$


The generalization is however a little weaker at one point. In Theorem 4 the thresholds $2 k \leq n_{1}, 2 \ell \leq n_{2}$ for validity are natural. If either $n_{1}$ or $n_{2}$ is smaller then the problem becomes trivial, all such sets can be selected in $\mathcal{F}$. In the generalization below there is no such natural threshold. There will be another difference in the formulation. We give the construction of the extremal family rather than the maximum number of sets.

Theorem 6. [13] Let $X_{1}, X_{2}$ be two disjoint sets of $n_{1}$ and $n_{2}$ elements, respectively. Some positive integers $k_{i}, \ell_{i}(1 \leq i \leq m)$ are given. Define $b=\max _{i}\left\{k_{i}, \ell_{i}\right\}$. Suppose that $9 b^{2} \leq n_{1}, n_{2}$. If $\mathcal{F}$ is an intersecting subfamily of

$$
\bigcup_{i=1}^{m}\left(\begin{array}{c}
X_{1}, X_{2} \\
k_{i}, \ell_{i}
\end{array}\right)
$$

then $|\mathcal{F}|$ cannot exceed the size of the largest trivially intersecting subfamily.

Sketch of the proof. The proof uses the so called cycle method used in a simple proof of Theorem 1 (see [12]). Its basic idea is to find the largest family of intersecting intervals of length $k$ along a cycle of length $n$ and then a simple double counting leads to the statement of the theorem. It is convenient to consider the cycle as $\mathbb{Z}_{n}$ and an interval as a set $\{i, i+$ $1, \ldots, i+k-1\} \bmod k$. It is easy to prove that the largest intersecting family of such intervals is trivially intersecting.

In the present proof cyclic permutation will be replaced by a product of two cyclic permutations. In notation: $\mathbb{Z}_{n_{1}} \times \mathbb{Z}_{n_{2}}$. Of course intervals will be replaced by direct products of intervals of length $k_{i}$ and $\ell_{i}$, that is by $k_{i} \times \ell_{i}$ rectangles. The "intersecting condition" is that any two rectangles must meet in one of the coordinates. More precisely, if the two rectangles are $\left\{i_{1}, i_{1}+1, \ldots, i_{1}+k_{u}-1\right\} \times\left\{i_{2}, i_{2}+1, \ldots, i_{2}+\ell_{u}-1\right\}$ and $\left\{j_{1}, j_{1}+\right.$ $\left.1, \ldots, j_{1}+k_{v}-1\right\} \times\left\{j_{2}, j_{2}+1, \ldots, j_{2}+\ell_{v}-1\right\}$ then either $\left\{i_{1}, i_{1}+1, \ldots, i_{1}+\right.$ $\left.k_{u}-1\right\} \cap\left\{j_{1}, j_{1}+1, \ldots, j_{1}+k_{v}-1\right\}$ or $\left\{i_{2}, i_{2}+1, \ldots, i_{2}+\ell_{u}-1\right\} \cap\left\{j_{2}, j_{2}+\right.$ $\left.1, \ldots, j_{2}+\ell_{v}-1\right\}$ is non-empty. We call a pair of rectangles having this property proj-intersecting. 
Let $\mathcal{R}_{i}$ be a family of $k_{i} \times \ell_{i}$ rectangles in $\mathbb{Z}_{n_{1}} \times \mathbb{Z}_{n_{2}}(1 \leq i \leq m)$. We say that $\mathcal{R}=\bigcup_{i=1}^{m} \mathcal{R}_{i}$ is a proj-intersecting family if, any two members are proj-intersecting.

One can prove the statement analogous to the theorem for the rectangles, that is, the largest $\mathcal{R}$ is trivially intersecting (if $n_{1}$ and $n_{2}$ are large) either in the projections in $\mathbb{Z}_{n_{1}}$ or in the projections in $\mathbb{Z}_{n_{2}}$.

In other words

$$
\sum_{i=1}^{m}\left|\mathcal{R}_{i}\right| \leq \max \left\{n_{1} \sum_{i=1}^{m} \ell_{i}, n_{2} \sum_{i=1}^{m} k_{i}\right\}
$$

holds. However this is not sufficient for the proof of the theorem. A weighted version is needed.

Lemma 1. Suppose that the positive integers $k_{i}, \ell_{i}, b, n_{1}, n_{2}$ satisfy the inequalities $k_{i}, \ell_{i} \leq b(1 \leq i \leq m), 9 b^{2}<n_{1}, n_{2}$. Let $\mathcal{R}_{i}$ be a family of $k_{i} \times \ell_{i}$ rectangles in $\mathbb{Z}_{n_{1}} \times \mathbb{Z}_{n_{2}}(1 \leq i \leq m)$. Suppose that $\mathcal{R}=\bigcup_{i=1}^{m} \mathcal{R}_{i}$ is a proj-intersecting family. Let $\lambda_{i}>0(1 \leq i \leq m)$ be real numbers. Then

$$
\sum_{i=1}^{m} \lambda_{i}\left|\mathcal{R}_{i}\right| \leq \max \left\{n_{1} \sum_{i=1}^{m} \lambda_{i} \ell_{i}, n_{2} \sum_{i=1}^{m} \lambda_{i} k_{i}\right\}
$$

holds.

Define the families

$$
\mathcal{F}_{i}=\left\{F \in \mathcal{F}:\left|F \cap X_{1}\right|=k_{i},\left|F \cap X_{2}\right|=\ell_{i}\right\} .
$$

We use double counting for the sum

$$
\sum_{F, \mathcal{C}_{1}, \mathcal{C}_{2}} s(F)
$$

where $\mathcal{C}_{j}$ is a cyclic permutation of $\mathbb{Z}_{n_{j}}(j=1,2), F \in \mathcal{F}$ and it forms a rectangle for the product of these two cyclic permutations and the weight $s(F)$ is defined in the following way:

$$
s(F)=s_{i}(F)=\frac{1}{n_{1} !} \cdot \frac{1}{n_{2} !}\left(\begin{array}{c}
n_{1} \\
k_{i}
\end{array}\right)\left(\begin{array}{c}
n_{2} \\
\ell_{i}
\end{array}\right) \text { if } F \in \mathcal{F}_{i} .
$$

Some tedious calculations and the usage of the lemma leads to the proof of the theorem. 


\section{A small detour: shadows}

Let $\mathcal{F} \subset\left(\begin{array}{c}{[n]} \\ k\end{array}\right)$ be a family of $k$-element subsets of $[n]$. Its shadow is defined as

$$
\sigma(\mathcal{F})=\{G:|G|=k-1, G \subset F \text { for some } F \in \mathcal{F}\} .
$$

The shadow problem is the following: given $n, k$ and $|\mathcal{F}|$, minimize $|\sigma(\mathcal{F})|$. It is obvious to believe that if we are lucky and $|\mathcal{F}|=\left(\begin{array}{l}a \\ k\end{array}\right)$ holds for an integer $a$ then the best construction is "to push all these $k$-element subsets into the corner" that is to take all $k$-element subsets of an $a$-element set $A$. Then the size of the shadow will be $\left(\begin{array}{c}a \\ k-1\end{array}\right)$.

This is really true and this pattern can be continued using the following lemma.

Lemma 2. [15], [11] If $0<k, m$ are integers then one can find integers $a_{k}>a_{k-1}>\ldots>a_{t} \geq t \geq 1$ such that

$$
m=\left(\begin{array}{c}
a_{k} \\
k
\end{array}\right)+\left(\begin{array}{c}
a_{k-1} \\
k-1
\end{array}\right)+\ldots+\left(\begin{array}{c}
a_{t} \\
t
\end{array}\right)
$$

and they are unique.

This is called the canonical form of $m$. Now we can formulate the solution to the shadow problem.

Theorem 7. (Shadow Theorem) [15], [11] If $n, k$ and $|\mathcal{F}|$ are given, the canonical form of $|\mathcal{F}|$ is

$$
|\mathcal{F}|=\left(\begin{array}{c}
a_{k} \\
k
\end{array}\right)+\left(\begin{array}{c}
a_{k-1} \\
k-1
\end{array}\right)+\ldots+\left(\begin{array}{c}
a_{t} \\
t
\end{array}\right)
$$

then

$$
|\sigma(\mathcal{F})| \geq\left(\begin{array}{c}
a_{k} \\
k-1
\end{array}\right)+\left(\begin{array}{c}
a_{k-1} \\
k-2
\end{array}\right)+\ldots+\left(\begin{array}{c}
a_{t} \\
t-1
\end{array}\right)
$$

and this bound is sharp.

We might also want to minimize the "deeper" shadow, the so called s-shadow: $\sigma_{s}(\mathcal{F})=\{G:|G|=k-s, G \subset F$ for some $F \in \mathcal{F}\}$. Theorem 6 can be formulated in this general form. 
Theorem 8. (Shadow Theorem) [15], [11] If $n, k$ and $|\mathcal{F}|$ are given, the canonical form of $|\mathcal{F}|$ is

$$
|\mathcal{F}|=\left(\begin{array}{c}
a_{k} \\
k
\end{array}\right)+\left(\begin{array}{c}
a_{k-1} \\
k-1
\end{array}\right)+\ldots+\left(\begin{array}{c}
a_{t} \\
t
\end{array}\right)
$$

then

$$
\left|\sigma_{s}(\mathcal{F})\right| \geq\left(\begin{array}{c}
a_{k} \\
k-s
\end{array}\right)+\left(\begin{array}{c}
a_{k-s} \\
k-1-s
\end{array}\right)+\ldots+\left(\begin{array}{c}
a_{t} \\
t-s
\end{array}\right)
$$

and this bound is sharp.

Lovász [17] found an estimate which is not sharp in most cases but is easier to handle. We need to generalize the binomial coefficients for real numbers. If $x$ is a real number, $\left(\begin{array}{l}x \\ k\end{array}\right)=\frac{x(x-1) \ldots(x-k+1)}{k !}$.

Theorem 9. (Lovász' version of the Shadow theorem) [17] If $\mathcal{A}$ is a family of k-element sets,

$$
|\mathcal{A}|=\left(\begin{array}{l}
x \\
k
\end{array}\right)
$$

then

$$
\left|\sigma_{s}(\mathcal{A})\right| \geq\left(\begin{array}{c}
x \\
k-s
\end{array}\right) .
$$

This estimate is sharp only when $x$ is an integer.

Daykin [3] noticed that the shadow theorem implies the Erdös-Ko-Rado theorem.

Proof. Let $\mathcal{F} \subset\left(\begin{array}{c}{[n]} \\ k\end{array}\right)$ be intersecting $(2 k \leq n)$. Define the complementing family $\mathcal{F}^{-}=\{[n]-F: F \in \mathcal{F}\} \subset\left(\begin{array}{c}{[n]} \\ n-k\end{array}\right)$ where $k \leq n-k$. If $A \in \mathcal{F}$ then $\bar{A} \in \mathcal{F}^{-}$has $n-k$ elements. Deleting $s=n-2 k$ elements from the $n-k$-element set $\bar{A}$ we obtain a $k$-element shadow set. Hence $\sigma_{n-2 k}\left(\mathcal{F}^{-}\right) \subset\left(\begin{array}{c}{[n]} \\ k\end{array}\right)$. The members of $\sigma_{n-2 k}\left(\mathcal{F}^{-}\right)$are all disjoint to $A$ therefore they cannot be in $\mathcal{F}$. We obtained

$$
\mathcal{F} \cap \sigma_{n-2 k}\left(\mathcal{F}^{-}\right)=\emptyset
$$

Suppose $\left|\mathcal{F}^{-}\right|=|\mathcal{F}|>\left(\begin{array}{l}n-1 \\ k-1\end{array}\right)=\left(\begin{array}{l}n-1 \\ n-k\end{array}\right)$. Then by Theorem $7\left|\sigma_{n-2 k}\left(\mathcal{F}^{-}\right)\right| \geq$ $\left(\begin{array}{c}n-1 \\ k\end{array}\right)$ and by $(2)$, the number of $k$-element subsets is at least $|\mathcal{F}|+$ $\left|\sigma_{n-2 k}\left(\mathcal{F}^{-}\right)\right|>\left(\begin{array}{c}n-1 \\ k-1\end{array}\right)+\left(\begin{array}{c}n-1 \\ k\end{array}\right)=\left(\begin{array}{l}n \\ k\end{array}\right)$. This contradiction proves the statement. 


\section{Shadows of intersecting families}

Suppose $\mathcal{F}$ is intersecting and $|\mathcal{F}|=\left(\begin{array}{l}a \\ k\end{array}\right)$ where $2 k<a<n$. If we want to find the minimum of $|\sigma(\mathcal{F})|$ under these conditions, then it is easy to see that the old construction does not work here since one cannot choose all $k$-element sets of the $a$-element set, since there are disjoint ones among them.

Let us consider the following more general case. $\mathcal{F}$ is $t$-intersecting if $F, G \in \mathcal{F}$ implies $|F \cap G| \geq t$. Our question is, again what is the minimum of $\left|\sigma_{s}(\mathcal{F})\right|$ under the condition that $\mathcal{F}$ is $t$-intersecting?

The disappointing answer is that we do not know! This is why we must ask a more modest question. What is the minimum of

$$
\frac{\left|\sigma_{s}(\mathcal{F})\right|}{|\mathcal{F}|}
$$

under the condition that $\mathcal{F}$ is $t$-intersecting?

Theorem 10. (Intersecting shadow theorem) [10] If $\mathcal{F} \subset\left(\begin{array}{c}{[n]} \\ k\end{array}\right)$ is a $t$-intersecting family, $s \leq t$ then

$$
\frac{\left|\sigma_{s}(\mathcal{F})\right|}{|\mathcal{F}|} \geq \frac{\left(\begin{array}{c}
2 k-t \\
k-s
\end{array}\right)}{\left(\begin{array}{c}
2 k-t \\
k
\end{array}\right)} .
$$

The family $\mathcal{F}=\left(\begin{array}{c}2 k-t \\ k\end{array}\right)$ gives equality in the theorem.

Now we will show that the Intersecting shadow theorem implies EKR. This has an importance because it is a less difficult theorem than the Shadow theorem, yet it has the same implication at this place.

Proof ([10]). We will start in the same way as in the proof of Daykin. (Observe that [10] was published earlier than [3].) As before let $\mathcal{F} \subset\left(\begin{array}{c}{[n]} \\ k\end{array}\right)$ be intersecting $(2 k \leq n)$ and $\mathcal{F}^{-}=\{[n]-F: F \in \mathcal{F}\} \subset\left(\begin{array}{c}{[n]} \\ n-k\end{array}\right)$ where $k \leq n-k$. We saw that $(*)$ holds.

$\mathcal{F}$ is intersecting therefore $\mathcal{F}^{-}=\{[n]-F: F \in \mathcal{F}\} \subset\left(\begin{array}{c}{[n]} \\ n-k\end{array}\right)$ is $n-2 k+1$ intersecting. Here $2(n-k)-(n-2 k+1)=n-1$ and by the intersecting shadow theorem we obtain

$$
\frac{\left|\sigma_{s}\left(\mathcal{F}^{-}\right)\right|}{\left|\mathcal{F}^{-}\right|} \geq \frac{\left(\begin{array}{c}
n-1 \\
k
\end{array}\right)}{\left(\begin{array}{c}
n-1 \\
n-k
\end{array}\right)}=\frac{n-k}{k} .
$$


Hence by $(*)$ :

$$
\left(\begin{array}{l}
n \\
k
\end{array}\right) \geq\left|\sigma_{s}\left(\mathcal{F}^{-}\right)\right|+|\mathcal{F}| \geq|\mathcal{F}|\left(\frac{n-k}{k}+1\right)=|\mathcal{F}| \frac{n}{k},
$$

which implies EKR.

Return now to Theorem 10. The problem answered by it is not just for itself. The solution of the maximization of the non-uniform $t$-intersecting family was based on that (see [10]). Repeat the result of Theorem 10 for the case $s=1$.

$$
\frac{|\sigma(\mathcal{F})|}{|\mathcal{F}|} \geq \frac{\left(\begin{array}{c}
2 k-t \\
k-1
\end{array}\right)}{\left(\begin{array}{c}
2 k-t \\
k
\end{array}\right)}=\frac{k-1}{k-t+1} .
$$

It was mentioned above that this estimate is sharp. If $\mathcal{F}$ consists of all $k$-element subsets of a $2 k-t$-element set then the size of the shadow is $\left(\begin{array}{c}2 k-t \\ k-1\end{array}\right)$, the ratio is exactly the above one. In this construction however the size $|\mathcal{F}|$ of the family is "small", does not depend on $n$. What happens if we suppose that $|\mathcal{F}|$ is large? We have a slight improvement in this case.

Theorem 11. [7]. If $\mathcal{F} \subset\left(\begin{array}{c}{[n]} \\ k\end{array}\right)$ is a t-intersecting family, $1 \leq t$ then

$$
|\sigma(\mathcal{F})| \geq|\mathcal{F}| \frac{k-1}{k-t}-c(k, t)
$$

where $c(k, t)$ does not depend on $n$ and $|\mathcal{F}|$.

This is an improvement only when $t>1$. A better multiplicative constant cannot be expected as the following example shows.

Divide $[n]$ into two parts, $X_{1}, X_{2}$ where $\left|X_{1}\right|=2 k-t-2,\left|X_{2}\right|=n-$ $2 k+t+2$ and define $\mathcal{F}$ as the family of all $k$-element sets $F$ such that $\left|F \cap X_{1}\right|=k-1,\left|F \cap X_{2}\right|=1$. Here $|\mathcal{F}|=\left(\begin{array}{c}2 k-t-2 \\ k-1\end{array}\right)(n-2 k+t+2),|\sigma(\mathcal{F})|=$ $\left(\begin{array}{c}2 k-t-2 \\ k-2\end{array}\right)(n-2 k+t+2)+\left(\begin{array}{c}2 k-t-2 \\ k-1\end{array}\right)$. Their ratio tends to $\frac{k-1}{k-t}$.

Let us remark that a similar statement can be found in the survey paper [5].

Remark 1. The constant in Theorem 11 can be explicitly given:

$$
c(k, t)=\frac{t-1}{(k-t)(k-t+1)}\left(\begin{array}{c}
2 k-t \\
k
\end{array}\right) .
$$


Our example above shows that a better multiplicative constant cannot be expected in Theorem 11. However, observe that the size of the family in the example is linear as a function of $n$. What happens if the size of the family grows faster? This question is answered in the following theorem.

Theorem 12. Suppose that $1 \leq t \leq k \leq n$ and choose an integer parameter $1 \leq u \leq k-t$. If $\mathcal{F} \subset\left(\begin{array}{c}{[n]} \\ k\end{array}\right)$ is a t-intersecting family, then

$$
\begin{array}{r}
|\sigma(\mathcal{F})| \geq|\mathcal{F}| \frac{k-u}{k-u-t+1}-\left(\begin{array}{c}
n \\
u-1
\end{array}\right) \frac{t-1}{(k-u-t+1)(k-u-t+2)}\left(\begin{array}{c}
2 k-t \\
k-u+1
\end{array}\right) \\
-\left(\begin{array}{c}
n \\
u-2
\end{array}\right) \frac{2(t-1)}{(k-u-t+1)(k-u-t+3)}\left(\begin{array}{c}
2 k-t \\
k-u+2
\end{array}\right) \\
\vdots \\
-\left(\begin{array}{c}
n \\
1
\end{array}\right) \frac{(u-1)(t-1)}{(k-u-t+1)(k-t)}\left(\begin{array}{c}
2 k-t \\
k-1
\end{array}\right) \\
-\left(\begin{array}{c}
n \\
0
\end{array}\right) \frac{u(t-1)}{(k-u-t+1)(k-t+1)}\left(\begin{array}{c}
2 k-t \\
k
\end{array}\right) .
\end{array}
$$

The order of magnitude of the "error terms" is $n^{u-1}$ therefore the statement is interesting only if $|\mathcal{F}|$ is larger. But then the limit of $\frac{|\sigma(\mathcal{F})|}{|\mathcal{F}|}$ is $\frac{k-u}{k-u-t+1}$ as $n$ tends to infinity.

The two extreme cases are of special interest. For $u=1$ Theorem 12 gives back Theorem 11. The case $u=k-t$ is formulated as a separate statement.

Corollary 1. If $\mathcal{F} \subset\left(\begin{array}{c}{[n]} \\ k\end{array}\right)$ is a t-intersecting family, $1 \leq t$ then

$$
|\sigma(\mathcal{F})| \geq t|\mathcal{F}|-O\left(n^{k-t-1}\right) .
$$

This statement is true for any size of $\mathcal{F}$, but it is void when its order of magnitude is not more than $n^{t-k-1}$.

Theorem 10 was actually stated for these $s$-shadows in [10], similarly, our Theorem 12 can be extended for this case, too. It is a really horrible formula, see [7]. 


\section{References}

[1] Martin Aigner and Günter M. Ziegler, Proofs from THE BOOK, Springer-Verlag, Berlin-Heidelberg, 1998.

[2] Noga Alon and Joel H. Spencer, The probabilistic method, Wiley Interscience Series in Discrete Mathematics and Optimization, John Wiley \& Sons, Inc. New York, 1992.

[3] D.E. Daykin, Erdös-Ko-Rado from Kruskal-Katona, J. Combin. Theory Ser A 17 (1974), 254-255.

[4] P. Erdős, Chao Ko, R. Rado, Intersection theorems for systems of finite sets, Quarterly J. of Math. (Oxford), Second Series 12 (1961), 313-320.

[5] Peter Frankl, The shifting technique in extremal set theory, Surveys in combinatorics 123 (1987), 81-110.

[6] P. Frankl, An Erdős-Ko-Rado Theorem for Direct Products, Europ. J. Combinatorics 17 (1996), 727-730.

[7] P. Frankl and Gyula O.H. Katona, Results on the shadow of intersecting families, in preparation.

[8] Dániel Gerbner and Balázs Patkós, Extremal Finite Set Theory, CRC Press, 2018.

[9] A.J.W. Hilton E.C. and Milner, Some intersection theorems for systems of finite sets, Quarterly J. of Math. (Oxford) 18 (1967), 369-384.

[10] G. Katona, Intersection theorems for systems of finite sets, Acta Math. Acad. Sci. Hungar. 15 (1964), 329-337.

[11] G. Katona, A theorem on finite sets, Theory of Graphs, Proc. Coll. held at Tihany, 1966, Akadémiai Kiadó, 187-207. 
[12] G.O.H. Katona, A simple proof of the Erdös-Chao Ko-Rado theorem, J. Combin. Theory Ser. B 13 (1972), 183-184.

[13] Gyula O.H. Katona, A general 2-part Erdős-Ko-Rado theorem, Opuscula Mathematica 37 (2017), no. 4, 577-588.

[14] Gyula O.H. Katona, New results on intersecting families of subsets, Proc. of the International Conference on Discrete Mathematics, 2018, Salem, Tamil Nadu, India, accepted.

[15] J.B. Kruskal, The number of simplices in a complex, Mathematical Optimization Techniques, University of California, (1963), 251-278.

[16] Metthew Kwan, Benny Sudakov, Pedro Vieira, Non-trivially intersecting multi-part families, J. Combin. Theory Ser A 156 (2018), 44-60.

[17] László Lovász Combinatorial Problems and Exercises, Akadémiai Kiadó, 1979. Problem 11.14.

Gyula O.H. Katona

Rényi Institute

Hungarian Academy of Sciences

Budapest, Hungary

ohkatona@renyi.hu 